\title{
Associations between Early Midlife Lifestyle Behaviors, Young Adult Cognitive Reserve and Advanced Predicted Brain Age in Late Midlife
}

Carol E. Franz, $\mathrm{PhD}^{1,2}$; Sean N. Hatton, $\mathrm{PhD}^{3}$; Jeremy A. Elman, $\mathrm{PhD}^{1,2}$; Teresa Warren ${ }^{4}$; Nathan A. Gillespie, PhD ${ }^{5,6}$; Nathan A. Whitsel, BS ${ }^{1,2}$; Olivia K. Puckett, BS ${ }^{1,2}$; Anders M. Dale, $\mathrm{PhD}^{3}$; Lisa T. Eyler, $\mathrm{PhD}^{1,2}$; Christine Fennema-Notestine, $\mathrm{PhD}^{1,2,3}$; Donald J. Hagler Jr., PhD ${ }^{3}$; Richard L. Hauger, $\mathrm{MD}^{1,2,12}$; Ruth McKenzie, $\mathrm{PhD}^{7}$; Michael C. Neale, $\mathrm{PhD}^{5}$; Matthew S.

Panizzon, $\mathrm{PhD}^{1,2}$; Rahul C. Pearce ${ }^{1,2}$; Chandra A. Reynolds, $\mathrm{PhD}^{8}$; Mark Sanderson-Cimino, $\mathrm{PhD}^{1,2,9}$; Rosemary Toomey, $\mathrm{PhD}^{7}$; Xin M. Tu, $\mathrm{PhD}^{10}$; Hong Xian, $\mathrm{PhD}^{11}$; Michael J. Lyons, $\mathrm{PhD}^{7}$; William S. Kremen, $\mathrm{PhD}^{1,2,12 \text {, }}$

${ }^{1}$ Department of Psychiatry, University of California San Diego, San Diego, CA, USA

${ }^{2}$ Center for Behavior Genetics of Aging, University of California, San Diego, San Diego, CA, USA

${ }^{3}$ Department of Radiology, University of California San Diego, San Diego, CA, USA

${ }^{4}$ Department of Psychology, San Diego State University, San Diego, CA, USA

${ }^{5}$ Virginia Institute for Psychiatric and Behavior Genetics, Department of Psychiatry, Virginia Commonwealth University, Richmond, VA, USA

${ }^{6}$ QIMR Berghofer Medical Research Institute, Herston, Queensland 4006, Australia

${ }^{7}$ Department of Psychological and Brain Sciences, Boston University, Boston, MA, USA

${ }^{8}$ Department of Psychology, University of California, Riverside, Riverside, CA, USA

${ }^{9}$ San Diego State University/University of California San Diego Joint Doctoral Program in Clinical Psychology, San Diego, CA, USA

${ }^{10}$ Department of Family Medicine, University of California San Diego, San Diego, CA, USA

${ }^{11}$ Department of Epidemiology \& Biostatistics, St. Louis University, St. Louis, MO, USA

${ }^{12}$ Center of Excellence for Stress and Mental Health, VA San Diego Healthcare System, San Diego, CA, USA

Corresponding author: Carol E. Franz, PhD, University of California San Diego, Department of Psychiatry, 9500 Gilman Drive MC 0738, La Jolla, CA 92093; cfranz@,health.ucsd.edu

WORD COUNT: 2955 


\section{Key Points}

Question: Do modifiable lifestyle behaviors in early midlife predict later accelerated brain aging and is that association moderated by cognitive reserve?

Findings: A lifestyle composite of smoking, alcohol consumption and social engagement at age 41 was associated with estimated brain age in late midlife. There was a significant moderation effect whereby more unfavorable lifestyle behaviors predicted more advanced brain aging, but only in those with low-to-moderate cognitive reserve.

Meaning: Favorable lifestyle behaviors appear to be protective for brain integrity especially among those with lower cognitive reserve. Early midlife efforts at prevention could be prioritized among those with lower cognitive reserve. 


\begin{abstract}
Importance: Both cognitive reserve and modifiable lifestyle behaviors are associated with dementia risk. The effect of early lifestyle behaviors and cognitive reserve on late midlife brain aging could inform early identification and risk reduction of future dementia.

Objective: Determine associations of young adult cognitive reserve, early midlife lifestyle behaviors, and the reserve-by-lifestyle interaction on late midlife brain age. Examine the relationship between mild cognitive impairment (MCI) and brain age.

Design: Participants were from the nationally representative Vietnam Era Twin Study of Aging (VETSA). Cognitive reserve was assessed at median age 20 years (IQR=1.38) with the Armed Forces Qualification Test (AFQT). Lifestyle behaviors (smoking, alcohol consumption, and social engagement) were assessed at median age $41(\mathrm{IQR}=5.00)$. Structural brain imaging conducted at median age $69(\mathrm{IQR}=4.74)$ was used to construct predicted brain age difference scores $(\mathrm{PBAD}=$ chronological age minus predicted brain age) and MCI was ascertained.
\end{abstract}

Setting: In-person cognitive testing (ages 20 and 69); mailed survey (age 41); structural MRI, MCI diagnosis at University of California, San Diego (age 69).

Participants: 431 community-dwelling men.

Exposures: AFQT; self-reported health and lifestyle behaviors.

Main outcomes and measures: PBAD scores; MCI.

Results. In fully adjusted mixed linear models, age 20 cognitive reserve and the age 41 lifestyle composite were associated with age $69 \mathrm{PBAD}[\mathrm{t}(104)=2.62, \mathrm{p}=0.01,95 \% \mathrm{CI} 0.874,6.285$; $\mathrm{t}(104)=3.37, \mathrm{p}=0.001,95 \% \mathrm{CI} 0.583,2.249$ respectively] as was the reserve-by-lifestyle interaction [ $\mathrm{t}(104)=-2.29, \mathrm{p}=0.02,95 \% \mathrm{CI}-2.330,-0.167]$. Unfavorable lifestyle predicted more advanced brain age, but only for those with lower young adult cognitive reserve. The MCI group had more advanced brain age $(\mathrm{F}(2,130)=3.13 ; \mathrm{p}=0.05)$.

Conclusions and relevance. Favorable lifestyle behaviors promoted resistance to accelerated brain aging 3 decades later for those with lower young adult cognitive reserve. High reserve appeared to be protective regardless of lifestyle. The association with MCI suggests that advanced PBAD scores reflect poorer brain integrity, although it is unclear if PBAD is related to Alzheimer's dementia specifically. Lower cognitive reserve increases risk for dementia, but early lifestyle modification may promote healthier brain aging and dementia risk reduction, particularly in those with lower reserve.

Study Type. Cohort Study 


\section{Introduction}

With increasing numbers of individuals developing mild cognitive impairment (MCI) and Alzheimer's Disease (AD), early risk reduction and prevention efforts are viewed as important in reducing and delaying dementia onset. ${ }^{1-3}$ Major guidelines and reviews consistently associate modifiable lifestyle behaviors of physical activity, tobacco use, diet, alcohol consumption, and social engagement with dementia risk. ${ }^{1-9}$ The 2020 Lancet Commission report proposed that modification of lifestyle behaviors could reduce dementia incidence by as much as $35 \% .{ }^{1,2}$ Higher cognitive reserve — an individual's overall cognitive capacity—is also protective against decline and dementia. ${ }^{10-13}$ In recent years, the concept of biomarkers of "biological age" has gained traction ${ }^{14,15}$ and has been applied to models of brain aging. ${ }^{16,17}$ Advanced brain aging has been observed in both MCI and AD, and may serve as a risk indictor for dementia. ${ }^{18,19}$ Toward the goal of early intervention and risk reduction, the primary aim of this project was to investigate associations of early midlife lifestyle behaviors and young adult cognitive reserve, including a lifestyle-by-reserve interaction, with late midlife brain aging.

Machine learning algorithms that predict chronological age in large samples of typically aging adults are applied to magnetic resonance imaging (MRI) features to calculate predicted brain age, a global measure of brain integrity. ${ }^{17,18,20}$ Advanced or accelerated brain aging is inferred when predicted brain age is older than expected given one's chronological age.

Cognitive decline from childhood has been associated with advanced brain age in midlife. ${ }^{20,21}$ AD patients' brain age appeared older than their chronological age by about 10 years ${ }^{18}$ and brain age was more advanced in MCI cases who progressed to AD than those who did not. ${ }^{19}$ Among $\mathrm{AD}$ and $\mathrm{MCI}$ cases who progressed to $\mathrm{AD}, 3$-year longitudinal increases in predicted brain age were greater in $A P O E-\varepsilon 4$ carriers than non-carriers. ${ }^{22}$ 
Lifestyle findings consistently support a dose-dependent response in that co-occurrence of multiple risky lifestyle behaviors is associated with worse outcomes compared with single risks. ${ }^{5,9}$ Among adults ages 55-85, for example, a favorable lifestyle composite was associated with healthier brain characteristics. ${ }^{23}$ To date, several studies using predicted brain age measures have examined individual lifestyle behaviors, but few have examined lifestyle composites. ${ }^{17,24}$ Concurrent associations of older brain age with smoking and alcohol consumption, but not physical activity, have been observed in middle-aged adults. ${ }^{17,24,25}$ The effect of alcohol dependence on predicted brain age was much stronger in older adults (as much as 11.7 years older) compared with middle aged adults. ${ }^{26}$ Brain age was also significantly correlated with type 2 diabetes, smoking duration, alcohol consumption, and depression within-time in adults in their mid-60s. ${ }^{16,27}$ Adults who both drank and smoked had more advanced brain age than those with only one of these behaviors. ${ }^{28}$ These cross-sectional results suggest that favorable lifestyle factors may promote resistance to accelerated brain aging. Theoretically, having a younger, healthier brain at midlife could ultimately reduce or delay onset of MCI or AD and other dementias. ${ }^{13}$

Despite the documented importance of cognitive reserve ${ }^{13}$ studies with direct measures of early cognitive reserve are rare. However, these are important because they help to address the issue of reverse causation. Age 11 childhood cognitive reserve, controlling for smoking and socioeconomic status, was a risk factor for vascular dementia but not AD in a 1921 Scottish birth cohort ${ }^{10}$ and higher age 11 cognitive reserve was associated with thicker cortex at age 73 in a 1936 Scottish birth cohort. ${ }^{11}$ Worse cognitive and brain health at age three predicted more advanced brain age in adults at age 45 , and older brain age was correlated with signs of advanced aging such as poorer health and older facial features. ${ }^{21}$ Cognitive reserve, then, may also promote 


\section{COGNITIVE RESERVE, LIFESTYLE AND BRAIN AGING}

resistance to accelerated brain aging. However, to our knowledge, there are no studies examining lifestyle-by-cognitive reserve interactions on brain age.

In the present study, we hypothesized that having fewer favorable lifestyle behaviors or lower young adult cognitive reserve would be associated with more advanced brain aging. We also tested a lifestyle-by-cognitive reserve interaction and predicted that young adult cognitive reserve would moderate the association between lifestyle behaviors and brain age. Finally, we examined whether predicted brain age is an indicator of cognitive decline by evaluating associations between MCI and brain aging in late midlife.

\section{Methods}

\section{Participants}

Participants were community-dwelling non-patient men from the Vietnam Era Twin Study of Aging (VETSA). ${ }^{29}$ VETSA comprise a sample of male-male twins who are members of the nationally representative Vietnam Era Twin (VET) Registry. They are similar to American men in their age range with respect to health and lifestyle characteristics. ${ }^{30}$ The majority $(\sim 80 \%)$ reported no combat exposure. VETSA participants were randomly recruited from 3322 twin pairs in the VET Registry. ${ }^{31}$ Ages at the three different assessments are provided below. Average lifetime education was 13.98 (SD 2.07) years.

\section{Procedures}

Young Adult Cognitive Reserve (median age 20)

Participants completed the Armed Forces Qualification Test (AFQT) in person (median age $=19.51$ years; $\mathrm{IQR}=1.38$; range $=17-25 ; 1965-1975)$ during induction into the military. The AFQT is a standardized, validated 100-item multiple-choice paper-and-pencil test of general cognitive ability (median age $=19.51$ years; $\mathrm{IQR}=1.38$; range $=17-25) .{ }^{32}$ This test is highly 


\section{COGNITIVE RESERVE, LIFESTYLE AND BRAIN AGING}

correlated with other tests of GCA such as Wechsler Adult Intelligence Scale $(r=0.84) .{ }^{33}$

Average intelligence of participants was estimated at $105 .{ }^{33}$

Early Midlife Modifiable Lifestyle Behaviors (median age 41)

In 1990, in early midlife (median age $=41 ; \mathrm{IQR}=5.00$; range $=34-44$ ), participants completed a mailed health survey which queried their smoking, alcohol consumption, social engagement, physical activity and diet behaviors (see Supplemental eTable 1). ${ }^{34}$ Each modifiable lifestyle indicator was operationalized based on standard criteria and coded as favorable (1) or unfavorable (0). ${ }^{7,8}$

Smoking status was defined as current (unfavorable) versus never/former (favorable). Unfavorable alcohol consumption was defined as drinking $>2$ drinks per day across the past 14 days. Drinking $\leq 2$ drinks per day, former, or never drinking was scored as favorable. Social engagement was operationalized based on 6 measures: marriage (currently married/not married), involvement in church more than twice a month (yes/no), clubs/organizations (yes/no), number of close friends $(\geq 3 ;<3)$, number of close relatives $(\geq 3 ;<3)$, and number of close people connected with at least once a month $(\geq 3 ;<3) .{ }^{7}$ Social engagement scores of $0-2$ were categorized as unfavorable and 3-6 as favorable.

Favorable physical activity reflected engaging in moderate-to-vigorous activity at least 3 days per week; less activity was coded as unfavorable. Favorable diet was coded if a participant met recommendations for 3 of 5 food groups per week: fish, fruits and vegetables, dairy, red meat, and processed meat. Table 1 displays the proportion of favorable classifications for each indicator. Frequencies of the 5 modifiable lifestyle behaviors at age 41 ranged from a low of $53 \%$ who met criteria for a favorable diet to $87 \%$ who met criteria for favorable alcohol consumption. 
Late Midlife Data Collection (median age 69)

In-person data collection during late midlife (median age $=68.90$ years; $\mathrm{IQR}=4.74$; range= 61-72; 2016-2019), included a detailed medical history interview, socio-demographic data, indepth cognitive testing, and structural MRI for those who passed a standard MRI safety screening $(\mathrm{N}=481)$. More details on VETSA procedures are published elsewhere. ${ }^{35-37}$

MRI Acquisition and Processing (median age 69). T1-weighted images providing high anatomical detail were acquired on 2 GE 3T Discovery $750 \times$ scanners (GE Healthcare, Waukesha, WI, USA) with an 8-channel phased array head coil (scanner $1 \mathrm{~N}=336$, scanner 2 $\mathrm{N}=145$ ) at the University of California, San Diego. The T1 imaging protocol included a sagittal 3D fast-spoiled gradient echo T1-weighted image (echo time $=3.164 \mathrm{msec}$, repetition time $=8.084$ msec, inversion time $=600 \mathrm{msec}$, flip angle $=8^{\circ}$, pixel bandwidth $=244.141$, field of view $=25.6 \mathrm{~cm}$, frequency $=256$, phase $=192$, slices $=172$, slice thickness $=1.2 \mathrm{~mm}$ ). As described in prior work raw image files were processed using an in-house pipeline by the UCSD Center for Multimodal Imaging and Genetics. ${ }^{38}$ Data were qualitatively assessed and images with severe scanner artifacts or excessive head motion were rescanned where possible or excluded from analysis $(\sim 3 \%)$. T1-weighted structural images were corrected for gradient distortions ${ }^{39}$ and B1 field inhomogeneity. ${ }^{40}$ Subcortical segmentation and surface-based cortical parcellation were performed using FreeSurfer version 5.1 (surfer.nmr.mgh.harvard.edu) as previously described. ${ }^{41}$ Inaccuracies in automated segmentations were manually corrected by trained neuroimaging analysts. All images required some form of manual editing to ensure the correct classification of the pial and white matter surfaces, with particular attention given to the orbitofrontal cortex, the temporal lobes, meninges, and transverse and superior sagittal sinuses. Problematic 


\section{COGNITIVE RESERVE, LIFESTYLE AND BRAIN AGING}

segmentations/parcellations were reviewed for exclusion by consensus with four neuroimaging analysts.

Late Midlife Predicted Brain Age. We used the Brain-Age Regression Analysis and Computation Utility software BARACUS v0.9.4 $4^{20}$ (https://github.com/BIDS-Apps/BARACUS; https:// zenodo.org/record/826543\#.WjF5Ft-nE2x). BARACUS uses linear support vector regression models to predict brain age in adults derived from each individual's FreeSurfer statistics. ${ }^{15}$ Specifically, vertex-wise cortical metrics were derived from the fsaverage4 standard space for cortical thickness ( $\mathrm{n}=5124$ vertices) and surface area ( $\mathrm{n}=5124$ vertices), and subcortical segmentation metrics were derived from the aseg.stat file for subcortical volume ( $\mathrm{n}=66$ regions of interest). We used the BIDS-mode docker on Ubuntu 16.04 using the default database (Liem2016_OCI_norm), which is trained on 1166 independent subjects with no objective cognitive impairment (566 female/600 male, $20-80$ years). ${ }^{20}$ The predicted brain age difference score (PBAD) is calculated by subtracting predicted brain age (referred to as "stacked-anatomy" brain age in BARACUS) from chronological age. Therefore, a negative PBAD is indicative of brain age that is estimated to be older than one's chronological age. PBAD was adjusted, via regression, for scanner. One participant was excluded due to brain cancer and 2 due to brain damage of unknown origin.

Late Midlife Mild Cognitive Impairment (MCI). The Jak-Bondi approach was used to diagnose $\mathrm{MCI}^{42-44}$ based on 18 cognitive tests covering memory, executive function, attention, language, visuospatial ability, and processing speed. ${ }^{29}$ Criteria for impairment within a domain required performance on $2+$ tests that were each $>1.5$ SDs below age- and education-adjusted normative means. Cognitive scores were adjusted for young adult GCA in order to differentiate 
decline from lifelong poor performance. ${ }^{45}$ Scores were additionally adjusted for practice and attrition effects using the replacement-subjects method as described previously. ${ }^{46}$

\section{Covariates}

Ethnicity was defined as non-Hispanic White versus other. Early midlife (age 41) covariates included a cardiovascular index: total of yes/no responses to angina pectoris, congestive heart failure, coronary heart disease, damaged heart valves, heart attack/myocardial infarction, phlebitis/thrombophlebitis, and stroke. The respiratory index included asthma, chronic obstructive pulmonary disease, and emphysema. Liver disease index comprised alcoholic hepatitis, cirrhosis of the liver. One item addressed physician diagnosed depression (yes/no). Due to the low frequency of health problems, the final cardiovascular, respiratory, and liver disease indices are categorized as any disorders versus none. Hypertension and diabetes were coded based on the response to "Have you ever been told by a doctor that you had __." Obesity was coded as BMI $\geq 30$ based on self-reported height and weight. Self-reported stroke at age 69 was also included as a covariate. Frequencies of these health items and their correlations with key variables are shown in Supplemental eTables 2 and 3. APOE genotype was classified as having $\varepsilon 4+$ versus $\varepsilon 4-.{ }^{47}$ Some data were missing for $A P O E(\mathrm{~N}=63)$ since not all participants provided blood or were genotyped. Participants were excluded if they self-reported a history of seizures, multiple sclerosis, HIV, or schizophrenia $(\mathrm{N}=6)$, leaving a final $\mathrm{N}$ of 431 with complete PBAD, cognitive reserve, lifestyle, and demographic data.

\section{Statistical analysis}

In preliminary analyses, alcohol consumption, smoking, and social engagement were the only lifestyle behaviors associated with later PBAD at $\mathrm{p}<.10$ (Supplemental eTable 4). These 3 measures were summed to create a lifestyle composite (0-3). As seen in Table $1,51 \%$ of the 
sample met criteria for favorable ratings on all 3 behaviors. Table 2 shows associations between key variables and number of favorable lifestyle behaviors.

We conducted linear mixed models in SAS 9.4 using maximum likelihood methods. The basic multivariable model (Model 1) examined the main effects of young adult cognitive reserve and the early midlife lifestyle composite, as well as the cognitive reserve-by-lifestyle interaction on late midlife PBAD adjusting for age, ethnicity, and stroke. Analyses treated twins as individuals so familial relationship was accounted for by including family as a random effect. The full model (Model 2) additionally adjusted for early midlife cardiovascular problems, hypertension, obesity, diabetes, respiratory problems, liver disease, depression, $A P O E$ genotype, and height. The $\mathrm{N}$ in the full model is 354, primarily because of missing APOE data. All results are reported as Type III tests of fixed effects, two-tailed.

\section{Results}

In the basic multivariable model (Model 1; Table 3) young adult cognitive reserve [F $(1,141)=7.85, \mathrm{p}=.006]$, early midlife lifestyle composite $[\mathrm{F}(1,141)=12.93, \mathrm{p}<0.001]$, and the reserve-by-lifestyle interaction $[\mathrm{F}(1,141)=5.40, \mathrm{p}=0.022]$ were associated with late midlife PBAD. Participants with lower young adult cognitive reserve or fewer early midlife favorable lifestyle behaviors had a predicted brain age that was older than expected given their chronological age. These results remained after adjustment for all covariates (Table 3, Model 2): cognitive reserve $[\mathrm{F}(1,104)=6.88, \mathrm{p}=0.01)]$, lifestyle composite $[\mathrm{F}(1,104)=111.37, \mathrm{p}=0.001)]$, reserve-by-lifestyle interaction $[\mathrm{F}(1,104)=5.24, \mathrm{p}=0.02)]$. With the exception of age, no covariates were significant in the full model.

The reserve-by-lifestyle composite interaction for Model 2 is depicted in Figure 1. For ease of illustration, we created 2 lifestyle groups $(0,1=$ unfavorable; $1,2=$ favorable $)$. Higher 


\section{COGNITIVE RESERVE, LIFESTYLE AND BRAIN AGING}

young adult cognitive reserve ( $\sim 75 \mathrm{SD}$ above the sample mean) was protective against effects of unfavorable lifestyle on PBAD. For those with low-to-moderate young adult cognitive reserve, unfavorable lifestyles at median age 41 were associated with older predicted brain age compared to their counterparts with favorable lifestyles. The difference between these lifestyle groups tended to be greater with lower reserve.

Having an older than predicted brain age was associated with MCI at median age 69 (F $(2,130)=3.13 ; \mathrm{p}=0.05)$. The non-amnestic MCI group had older than predicted brain age $($ mean $\mathrm{PBAD}=-3.62, \mathrm{SE}=1.14)$ compared with the amnestic $\mathrm{MCI}$ group $($ mean PBAD $=$ $-0.61, \mathrm{SE}=1.06 ; \mathrm{t}=-2.31 ; \mathrm{p}=.02 ; 95 \% \mathrm{CI}-5.578,-0.431)$ or cognitively normal group (mean $\mathrm{PBAD}=-1.15, \mathrm{SE}=.73 ; \mathrm{t}=-2.37 ; \mathrm{p}=.02 ; 95 \% \mathrm{CI}-4.535,-0.406)$.

\section{Discussion}

Young adult cognitive reserve and modifiable lifestyle behaviors in early midlife predicted brain aging over a period spanning four decades. High cognitive reserve appeared to be protective regardless of lifestyle behaviors, but favorable lifestyle was protective for those with low-to-moderate cognitive reserve. Consistent with studies of much older adults, predicted brain age was associated with MCI. ${ }^{19,22}$ In those studies brain age also predicted conversion to AD. AD patients' brain age appears older than their chronological age by about 10 years. ${ }^{18}$ In the much younger VETSA sample, participants with non-amnestic MCI had older-than-predicted brains than either the amnestic MCI or cognitively normal groups. Favorable lifestyle behaviors such as not smoking or low alcohol consumption are presumed to contribute to global brain integrity and cognitive health through multiple pathways involving cardiovascular risk and neurotoxic effects ${ }^{48-52}$. Mechanisms behind social integration's benefits are less clear. ${ }^{6,53,54}$ These 
results suggest that unfavorable lifestyle behaviors as early as midlife increase risk for dementia, but it remains unclear of if the effect is related to AD per se.

Our study is unique in its examination of the cognitive reserve-by-lifestyle interaction. There is evidence that overall cognitive ability peaks in the 20 s-to-early 30 s, so these participants may have been close to peak reserve at the time of the AFQT assessment. ${ }^{35}$ In addition, the lifestyle composite data were collected earlier in the life course compared with most studies of long-term effects of lifestyle on the brain. ${ }^{1,2}$ These results may be thought of as indicating that, regardless of level of young adult cognitive reserve, favorable lifestyle confers resistance to advanced brain aging regardless; however, high reserve confers both resistance to advanced brain aging and resilience against the effects of toxic lifestyle behaviors. ${ }^{13,55}$ PBAD also suggests brain maintenance, ${ }^{56}$ but confirmation of brain maintenance requires longitudinal brain data.

Meta-analyses suggest that a brain health risk reduction agenda could be effective in reducing risk for dementia. ${ }^{2,3,57}$ Both the U.K. National Institute of Health and Care Excellence, and the U.S. National Institutes of Health list smoking and social integration as among the top modifiable risk factors for dementia. The 2020 Lancet Commission reported that a third of dementia incidence can be attributed to modifiable risk factors ${ }^{1}$, although this remains to be fully supported by clinical trials. ${ }^{58}$ Smoking, alcohol consumption, and social integration in the first half of the life cycle may be potential modifiable targets for early intervention for brain health. In one randomized controlled trial, for example, brain volume increased across 40 weeks in an intervention involving social interaction. ${ }^{54}$

There are several limitations to this study. Participants were all men and mostly nonHispanic White, thus results may not generalize across genders or different racial or ethnic 


\section{COGNITIVE RESERVE, LIFESTYLE AND BRAIN AGING}

origins. Lifestyle indicators were self-reported; however, that is typical for such measures. We also cannot definitively rule out whether PBAD was already different at the time lifestyle behaviors were assessed since we did not have a measure of predicted brain age at that time. Because patterns of atrophy for global brain aging might be different from the patterns associated with $\mathrm{AD},{ }^{59}$ further follow-up will be needed to determine the extent to which PBAD may be a longitudinal risk factor for $\mathrm{AD}$ or MCI.

\section{Conclusions}

Given the large number of persons likely to develop dementia in the next decades, early identification of modifiable risk factors may be key to identifying and reducing the incidence and burden of $\mathrm{AD}$ and other dementias. These results highlight the potential value of focusing on preventive efforts earlier in development and not just in later life. Given the evidence suggesting that favorable lifestyle confers resistance to advanced brain aging particularly among those with lower cognitive reserve, efforts at prevention could be prioritized among those with lower cognitive reserve. With increasing number of individuals with $\mathrm{MCI}$ and $\mathrm{AD}$ combined with a lack of effective treatments, early risk reduction and prevention can be major components in efforts to facilitate maintenance of brain integrity, thereby mitigating individual and societal health, economic, and social burden. 


\section{COGNITIVE RESERVE, LIFESTYLE AND BRAIN AGING}

\section{Funding}

This work was supported by the National Institute on Aging at the National Institutes of Health grant numbers R01s AG050595, AG022381, AG037985; R25 AG043364, F31 AG064834, and P01 AG055367.

\section{Acknowledgements}

The content is the responsibility of the authors and does not necessarily represent official views of the NIA, NIH, or VA. The U.S. Department of Veterans Affairs, Department of Defense; National Personnel Records Center, National Archives and Records Administration; National Opinion Research Center; National Research Council, National Academy of Sciences; and the Institute for Survey Research, Temple University provided invaluable assistance in the creation of the VET Registry. The Cooperative Studies Program of the U.S. Department of Veterans Affairs provided financial support for development and maintenance of the Vietnam Era Twin Registry. We would also like to acknowledge the continued cooperation and participation of the members of the VET Registry and their families. Ms. Warren was supported by the Advancing

Diversity in Aging Research (ADAR) program at San Diego State University (R25 AG043364). 


\section{COGNITIVE RESERVE, LIFESTYLE AND BRAIN AGING}

\section{References}

1. Livingston G, Huntley J, Sommerlad A, et al. Dementia prevention, intervention, and care: 2020 report of the Lancet Commission. Lancet. 2020;396(10248):413-446.

2. Orgeta V, Mukadam N, Sommerlad A, Livingston G. The Lancet Commission on Dementia Prevention, Intervention, and Care: a call for action. Ir J Psychol Med. 2019;36(2):85-88.

3. World Health Organization. Risk Reduction of Cognitive Decline and Dementia: WHO Guidelines. Geneva2019.

4. Anstey KJ, Cherbuin N, Herath PM, et al. A self-report risk index to predict occurrence of dementia in three independent cohorts of older adults: the ANU-ADRI. PLoS One. 2014;9(1):e86141.

5. Anstey KJ, Eramudugolla R, Dixon RA. Contributions of a risk assessment approach to the prevention of Alzheimer's disease and dementia. J Alzheimers Dis. 2014;42 Suppl 4:S463-473.

6. Fratiglioni L, Paillard-Borg S, Winblad B. An active and socially integrated lifestyle in late life might protect against dementia. Lancet Neurol. 2004;3(6):343-353.

7. Licher S, Ahmad S, Karamujic-Comic H, et al. Genetic predisposition, modifiable-riskfactor profile and long-term dementia risk in the general population. Nat Med. 2019;25(9):1364-1369.

8. Lourida I, Hannon E, Littlejohns TJ, et al. Association of Lifestyle and Genetic Risk With Incidence of Dementia. JAMA. 2019.

9. Peters R, Booth A, Rockwood K, Peters J, D'Este C, Anstey KJ. Combining modifiable risk factors and risk of dementia: a systematic review and meta-analysis. BMJ Open. 2019;9(1):e022846.

10. McGurn B, Deary IJ, Starr JM. Childhood cognitive ability and risk of late-onset Alzheimer and vascular dementia. Neurology. 2008;71(14):1051-1056. 


\section{COGNITIVE RESERVE, LIFESTYLE AND BRAIN AGING}

11. Karama S, Bastin ME, Murray C, et al. Childhood cognitive ability accounts for associations between cognitive ability and brain cortical thickness in old age. Mol Psychiatry. 2014;19(5):555-559.

12. Vuoksimaa E, Panizzon MS, Chen $\mathrm{CH}$, et al. Cognitive reserve moderates the association between hippocampal volume and episodic memory in middle age. Neuropsychologia. 2013;51(6):1124-1131.

13. Stern Y, Arenaza-Urquijo EM, Bartres-Faz D, et al. Whitepaper: Defining and investigating cognitive reserve, brain reserve, and brain maintenance. Alzheimers Dement. 2018.

14. Li X, Ploner A, Wang Y, et al. Longitudinal trajectories, correlations and mortality associations of nine biological ages across 20-years follow-up. Elife. 2020;9.

15. Bocklandt S, Lin W, Sehl ME, et al. Epigenetic predictor of age. PLoS One. 2011;6(6):e14821.

16. Franke K, Gaser C. Ten Years of BrainAGE as a Neuroimaging Biomarker of Brain Aging: What Insights Have We Gained? Front Neurol. 2019;10:789.

17. Cole JH, Marioni RE, Harris SE, Deary IJ. Brain age and other bodily 'ages': implications for neuropsychiatry. Mol Psychiatry. 2019;24(2):266-281.

18. Franke K, Ziegler G, Kloppel S, Gaser C, Alzheimer's Disease Neuroimaging I. Estimating the age of healthy subjects from T1-weighted MRI scans using kernel methods: exploring the influence of various parameters. Neuroimage. 2010;50(3):883892.

19. Gaser C, Franke K, Kloppel S, Koutsouleris N, Sauer H, Alzheimer's Disease Neuroimaging I. BrainAGE in Mild Cognitive Impaired Patients: Predicting the Conversion to Alzheimer's Disease. PLoS One. 2013;8(6):e67346.

20. Liem F, Varoquaux G, Kynast J, et al. Predicting brain-age from multimodal imaging data captures cognitive impairment. Neuroimage. 2017;148:179-188. 
COGNITIVE RESERVE, LIFESTYLE AND BRAIN AGING

21. Elliott ML, Belsky DW, Knodt AR, et al. Brain-age in midlife is associated with accelerated biological aging and cognitive decline in a longitudinal birth cohort. Mol Psychiatry. 2019.

22. Lowe LC, Gaser C, Franke K, Alzheimer's Disease Neuroimaging I. The Effect of the APOE Genotype on Individual BrainAGE in Normal Aging, Mild Cognitive Impairment, and Alzheimer's Disease. PLoS One. 2016;11(7):e0157514.

23. Bittner N, Jockwitz C, Muhleisen TW, et al. Combining lifestyle risks to disentangle brain structure and functional connectivity differences in older adults. Nat Commun. 2019;10(1):621.

24. Cole $\mathrm{JH}$. Multimodality neuroimaging brain-age in UK biobank: relationship to biomedical, lifestyle, and cognitive factors. Neurobiol Aging. 2020;92:34-42.

25. Hatton SN, Franz CE, Elman JA, et al. Negative fateful life events in midlife and advanced predicted brain aging. Neurobiol Aging. 2018;67:1-9.

26. Guggenmos M, Schmack K, Sekutowicz M, et al. Quantitative neurobiological evidence for accelerated brain aging in alcohol dependence. Transl Psychiatry. 2017;7(12):1279.

27. Franke K, Gaser C, Manor B, Novak V. Advanced BrainAGE in older adults with type 2 diabetes mellitus. Front Aging Neurosci. 2013;5:90.

28. Ning K, Zhao L, Matloff W, Sun F, Toga AW. Association of relative brain age with tobacco smoking, alcohol consumption, and genetic variants. Sci Rep. 2020;10(1):10.

29. Kremen WS, Franz CE, Lyons MJ. Current Status of the Vietnam Era Twin Study of Aging (VETSA). Twin Research and Human Genetics. 2020:1-5.

30. Centers for Disease Control and Prevention. Health data for all ages. National Center for Health Statistics;2007.

31. Eisen SA, True WR, Goldberg J, Henderson W, Robinette CD. The Vietnam Era Twin (VET) Registry: Method of construction. Acta geneticae medicae et gemellologiae (Roma). 1987;36:61-66. 


\section{COGNITIVE RESERVE, LIFESTYLE AND BRAIN AGING}

32. Uhlaner JE. Development of the Armed Forces Qualification Test and predecessor army screening tests, 1946-1950. PRB Rep. 1952.

33. Lyons MJ, York TP, Franz CE, et al. Genes determine stability and the environment determines change in cognitive ability during 35 years of adulthood. Psychol Sci. 2009;20(9):1146-1152.

34. Henderson WG, Eisen SE, Goldberg J, True WR, Barnes JE, Vitek M. The Vietnam Era Twin Registry: A resource for medical research. Public Health Rep. 1990;105:368-373.

35. Kremen WS, Beck A, Elman JA, et al. Influence of young adult cognitive ability and additional education on later-life cognition. Proc Natl Acad Sci U S A. 2019;116(6):20212026.

36. Kremen WS, Fennema-Notestine C, Eyler LT, et al. Genetics of brain structure: contributions from the Vietnam Era Twin Study of Aging. American Journal of Medical Genetics Part B Neuropsychiatric Genetics. 2013;162B(7):751-761.

37. Kremen WS, Thompson-Brenner H, Leung YM, et al. Genes, environment, and time: the Vietnam Era Twin Study of Aging (VETSA). Twin Research and Human Genetics. 2006;9(6):1009-1022.

38. Eyler LT, Chen $\mathrm{CH}$, Panizzon MS, et al. A comparison of heritability maps of cortical surface area and thickness and the influence of adjustment for whole brain measures: a magnetic resonance imaging twin study. Twin Research and Human Genetics. 2012;15(3):304-314.

39. Jovicich J, Czanner S, Greve D, et al. Reliability in multi-site structural MRI studies: Effects of gradient non-linearity correction on phantom and human data. Neuroimage. 2006;30:436-443.

40. Sled JG, Zijdenbos AP, Evans AC. A nonparametric method for automatic correction of intensity nonuniformity in MRI data. IEEE Trans Med Imaging. 1998;17:87-97.

41. Fischl B. FreeSurfer. Neuroimage. 2012;62(2):774-781. 


\section{COGNITIVE RESERVE, LIFESTYLE AND BRAIN AGING}

42. Jak AJ, Bondi MW, Delano-Wood L, et al. Quantification of five neuropsychological approaches to defining mild cognitive impairment. Am J Geriatr Psychiatry. 2009;17(5):368-375.

43. Kremen WS, Jak AJ, Panizzon MS, et al. Early identification and heritability of mild cognitive impairment. Int J Epidemiol. 2014;43(2):600-610.

44. Jak AJ, Panizzon MS, Spoon KM, et al. Hippocampal Atrophy Varies by Neuropsychologically Defined MCl Among Men in Their 50s. American Journal of Geriatric Psychiatry. 2015;23(5):456-465.

45. Lyons MJ, Panizzon MS, Liu W, et al. A Longitudinal Twin Study of General Cognitive Ability Over Four Decades. Dev Psychol. 2017.

46. Elman JA, Jak AJ, Panizzon MS, et al. Underdiagnosis of mild cognitive impairment: A consequence of ignoring practice effects. Alzheimers Dement (Amst). 2018;10:372-381.

47. Lyons MJ, Genderson M, Grant MD, et al. Gene-environment interaction of ApoE genotype and combat exposure on PTSD. Am J Med Genet B Neuropsychiatr Genet. 2013;162(7):762-769.

48. Cardenas VA, Hough CM, Durazzo TC, Meyerhoff DJ. Cerebellar Morphometry and Cognition in the Context of Chronic Alcohol Consumption and Cigarette Smoking. Alcohol Clin Exp Res. 2020;44(1):102-113.

49. Mazzone P, Tierney W, Hossain M, Puvenna V, Janigro D, Cucullo L. Pathophysiological impact of cigarette smoke exposure on the cerebrovascular system with a focus on the blood-brain barrier: expanding the awareness of smoking toxicity in an underappreciated area. Int J Environ Res Public Health. 2010;7(12):4111-4126.

50. Matloff WJ, Zhao L, Ning K, Conti DV, Toga AW. Interaction effect of alcohol consumption and Alzheimer disease polygenic risk score on the brain cortical thickness of cognitively normal subjects. Alcohol. 2020;85:1-12. 


\section{COGNITIVE RESERVE, LIFESTYLE AND BRAIN AGING}

51. Mende MA. Alcohol in the Aging Brain - The Interplay Between Alcohol Consumption, Cognitive Decline and the Cardiovascular System. Front Neurosci. 2019;13:713.

52. Starke RM, Thompson JW, Ali MS, et al. Cigarette Smoke Initiates Oxidative StressInduced Cellular Phenotypic Modulation Leading to Cerebral Aneurysm Pathogenesis. Arterioscler Thromb Vasc Biol. 2018;38(3):610-621.

53. Kelly ME, Duff H, Kelly S, et al. The impact of social activities, social networks, social support and social relationships on the cognitive functioning of healthy older adults: a systematic review. Syst Rev. 2017;6(1):259.

54. Mortimer JA, Ding D, Borenstein AR, et al. Changes in brain volume and cognition in a randomized trial of exercise and social interaction in a community-based sample of nondemented Chinese elders. J Alzheimers Dis. 2012;30(4):757-766.

55. Aganj I, Yeo BT, Sabuncu MR, Fischl B. On removing interpolation and resampling artifacts in rigid image registration. IEEE Trans Image Process. 2013;22(2):816-827.

56. Nyberg L, Lovden M, Riklund K, Lindenberger U, Backman L. Memory aging and brain maintenance. Trends Cogn Sci. 2012;16(5):292-305.

57. Hussenoeder FS, Riedel-Heller SG. Primary prevention of dementia: from modifiable risk factors to a public brain health agenda? Soc Psychiatry Psychiatr Epidemiol. 2018;53(12):1289-1301.

58. Montero-Odasso M, Ismail Z, Livingston G. One third of dementia cases can be prevented within the next 25 years by tackling risk factors. The case "for" and "against". Alzheimers Res Ther. 2020;12(1):81.

59. Habes M, Janowitz D, Erus G, et al. Advanced brain aging: relationship with epidemiologic and genetic risk factors, and overlap with Alzheimer disease atrophy patterns. Trans/ Psychiatry. 2016;6:e775. 


\section{COGNITIVE RESERVE, LIFESTYLE AND BRAIN AGING}

\section{Figure Legend}

Figure 1. Interaction between Young Adult Cognitive Reserve and Early Midlife Modifiable Lifestyle Behaviors on Predicted Brain Age Difference Scores (PBAD) in Late Midlife.

The reserve-by-lifestyle interaction is illustrated by categorizing lifestyle into two groups low $(0,1)$ versus more $(2,3)$ favorable lifestyle behaviors assessed at median age 41 .

Cognitive reserve is the continuous measure of cognitive ability assessed at median age 20. 
Table 1. Early Midlife Favorable Lifestyle Behaviors and Frequencies

Behavior

Definition of Favorable rating

$(0=$ unfavorable $/ 1=$ favorable $)$
N (\%) Favorable

Lifestyle
Alcohol Consumption

Tobacco Smoking

Social Engagement

Diet

Physical activity

Lifestyle Composite
Drinks $\leq 2$ drinks containing alcohol per day

Never or former

Engages in 3-6 forms of social interaction regularly

Consumes 3-5 Healthy food groups per day

Moderate to vigorous activity at least 3 days/week

Sum of alcohol consumption, smoking and social

engagement; higher score $=$ more favorable behaviors
$409(87.4 \%)$

$342(72.6 \%)$

$360(77.8 \%)$

$243(53.3 \%)$

$386(83.4 \%)$

$0=6(1.3 \%)$

$1=50(11.2 \%)$

$2=165(36.9 \%)$

$3=226(50.6 \%)$ 


\section{COGNITIVE RESERVE, LIFESTYLE AND BRAIN AGING}

Table 2. Basic Demographics and Descriptive Statistics

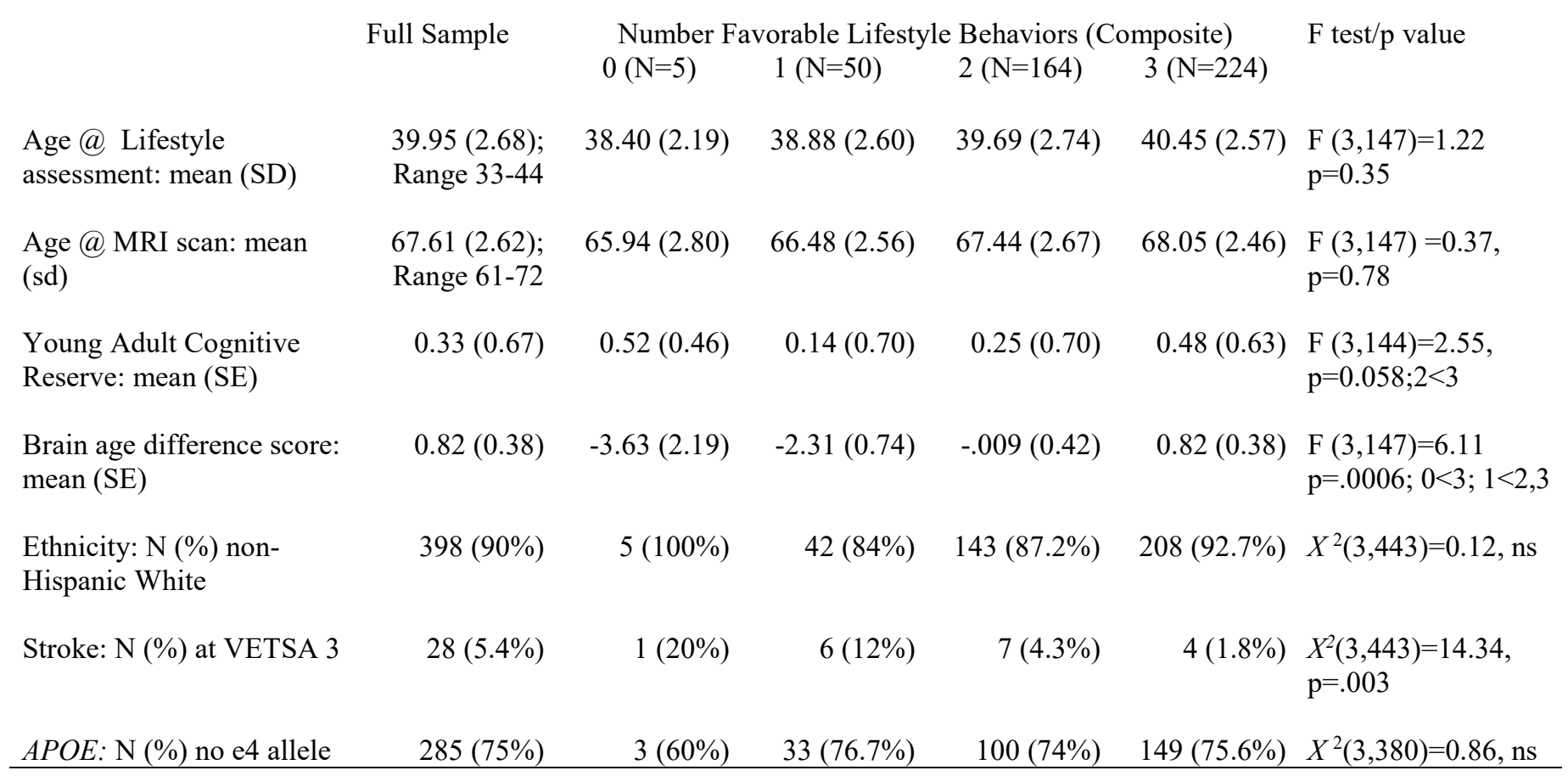

Table note: For $\mathrm{F}$ tests, $\mathrm{N}=$ range from 431 (age $20 \mathrm{AFQT}$ ) to 437 (others) but df is lower due to random effect of case. 
Table 3. Linear Mixed Effects Models Predicting the Brain Age Difference Score (PBAD) in Late Midlife.

\begin{tabular}{|c|c|c|c|c|c|c|c|c|}
\hline \multirow[b]{2}{*}{ Effect } & \multicolumn{4}{|c|}{ Basic Model (Model 1) } & \multicolumn{4}{|c|}{ Full Model (Model 2) } \\
\hline & $\begin{array}{l}\text { Estimate } \\
\text { (SE) }\end{array}$ & t Value & $\operatorname{Pr}>|t|$ & $\begin{array}{c}95 \% \mathrm{CI} \\
\text { (lower, upper) }\end{array}$ & $\begin{array}{l}\text { Estimate } \\
\text { (SE) }\end{array}$ & t Value & $\operatorname{Pr}>|t|$ & $\begin{array}{c}95 \% \mathrm{CI} \\
\text { (lower, upper) }\end{array}$ \\
\hline Intercept & $-33.82(7.48)$ & -4.52 & $<.0001$ & $-48.5342,-19.1022$ & $-46.34(12.05)$ & -3.85 & 0.0002 & $-70.0698,-22.6025$ \\
\hline $\begin{array}{l}\text { Cognitive reserve (Age } \\
20)\end{array}$ & $3.40(1.21)$ & 2.80 & 0.006 & $0.9995,5.7868$ & $3.57(1.36)$ & 2.62 & 0.010 & $0.8739,6.2847$ \\
\hline $\begin{array}{l}\text { Modifiable lifestyle } \\
\text { behaviors (Age 40) }\end{array}$ & $1.32(0.37)$ & 3.60 & 0.001 & $0.5929,2.0414$ & $1.42(0.42)$ & 3.37 & 0.001 & $0.5834,2.2488$ \\
\hline $\begin{array}{l}\text { Cognitive reserve by } \\
\text { lifestyle interaction }\end{array}$ & $-1.13(0.49)$ & -2.32 & 0.022 & $-2.0908,-0.1688$ & $-1.25(0.55)$ & -2.29 & 0.024 & $-2.3296,-0.1668$ \\
\hline $\begin{array}{l}\text { Ethnicity(Ref=non- } \\
\text { White) }\end{array}$ & $1.82(0.95)$ & 1.91 & 0.059 & $-0.06865,3.7065$ & $1.66(1.18)$ & 1.40 & 0.164 & $-0.6864,4.0050$ \\
\hline Age at MRI & $0.41(0.11)$ & 3.65 & 0.001 & $0.1860,0.6255$ & $0.45(0.13)$ & 3.56 & 0.001 & $0.1999,0.7019$ \\
\hline Stroke $($ Ref $=$ stroke $)$ & $1.60(1.15)$ & 1.39 & 0.167 & $-0.6762,3.8802$ & $0.96(1.40)$ & 0.69 & 0.494 & $-1.8200,3.7468$ \\
\hline \multicolumn{9}{|c|}{ Early Midlife Covariates: } \\
\hline Cardiovascular index & & & & & $-1.02(1.68)$ & -0.61 & 0.545 & $-4.3458,2.3099$ \\
\hline Obesity (BMI>=30) & & & & & $-0.64(1.24)$ & -0.51 & 0.609 & $-3.0935,1.8227$ \\
\hline Hypertension (yes/no) & & & & & $0.95(1.06)$ & 1.15 & 0.254 & $-0.6585,2.4688$ \\
\hline Diabetes (yes/no) & & & & & $0.56(2.79)$ & 0.20 & 0.840 & $-4.9616,6.0908$ \\
\hline Respiratory index & & & & & $-0.59(1.24)$ & -0.47 & 0.637 & $-3.0467,1.8710$ \\
\hline $\begin{array}{l}\text { Alcohol hepatitis/ } \\
\text { Cirrhosis (yes/no) }\end{array}$ & & & & & $1.48(4.66)$ & 0.32 & 0.752 & $-7.7669,10.7175$ \\
\hline Depression (yes/no) & & & & & $0.91(0.79)$ & 0.90 & 0.372 & $-1.1479,3.0402$ \\
\hline Height & & & & & $0.13(0.09)$ & 1.40 & 0.166 & $-0.05411,0.311$ \\
\hline$A P O E(\mathrm{Ref}=\mathrm{e} 4+)$ & & & & & $-0.42(0.71)$ & -0.60 & 0.552 & $-1.8375,0.9881$ \\
\hline
\end{tabular}

Abbreviations: $A P O E=$ Apolipoprotein $\mathrm{E}(\mathrm{e} 4+$ indicates presence of any e4 allele). Model $1 \mathrm{~N}=431 ; \mathrm{df}=1,141 ; \mathrm{Model} 2 \mathrm{~N}=354 ; \mathrm{df}=1,104(\mathrm{~N}$ lower due to missing covariates). All models adjust for the random effect of family. 
bioRxiv preprint doi: https://doi. org/10.1101/202011.02 362780. this version posted November 3,2020 . The copyright holder for this preprint (which was not certified by peer review) is the author/funder, who has granted bioRxiv a license to display the preprint in perpetuity. It is made available under aCC-BY-NC-ND 4.0 International license.

\section{Figure 1}

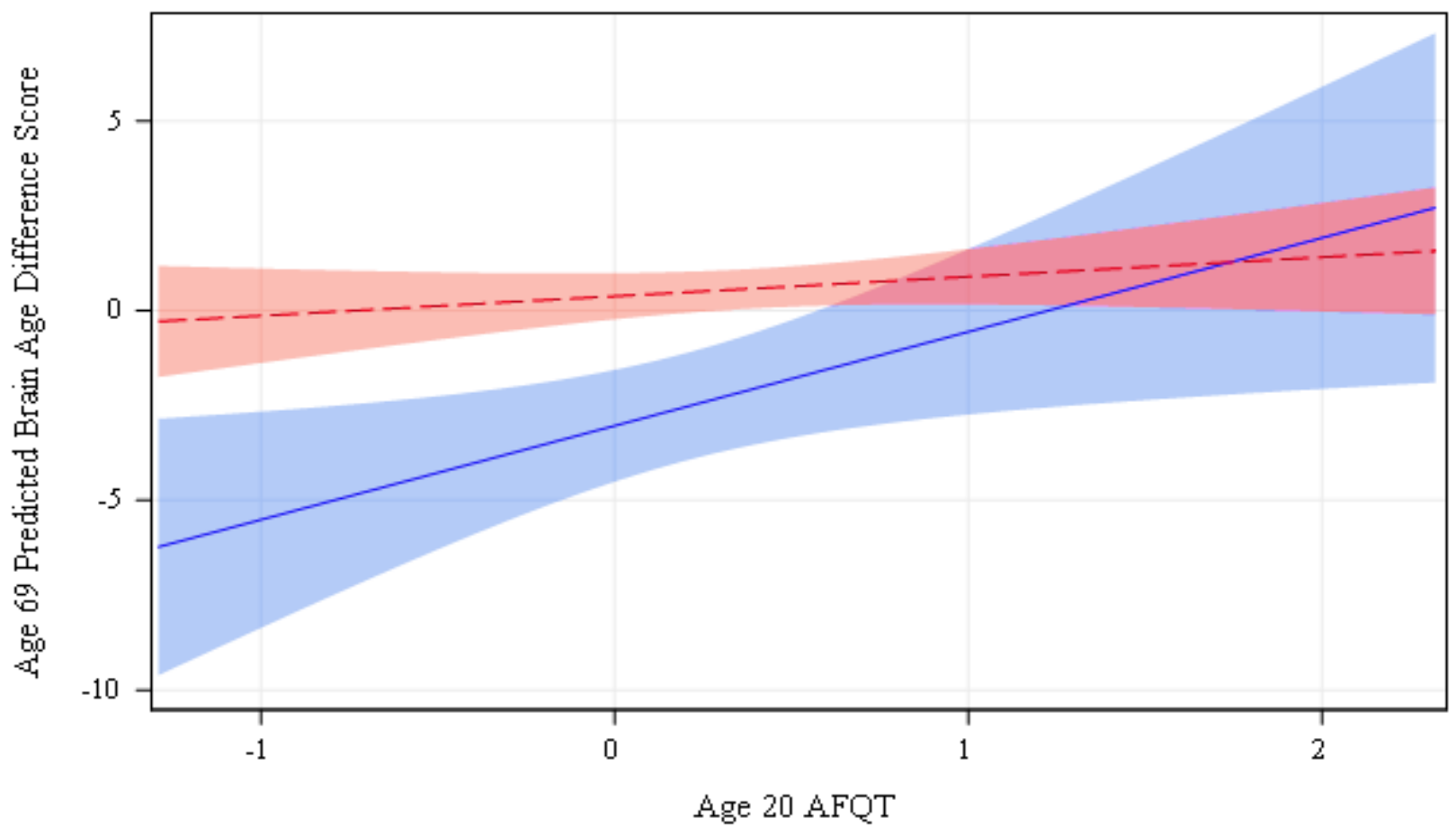

Few (0,1) versus More (2,3) Favorable Lifestyle Behaviors $\quad-0---1$ 
bioRxiv preprint doi: https://doi.org/10.1101/202011.02 362780; this version posted November 3, 2020. The copyright holder for this preprint

(which was not certified by peer review) is the author/funder, who has granted bioRxiv a license to display the preprint in perpetuity. It is made available under aCC-BY-NC-ND 4.0 International license.

\section{Associations between Early Midlife Lifestyle Behaviors, Young Adult Cognitive Reserve and Advanced Predicted Brain Age in Late Midlife}

Online supplemental tables 


\section{eTable 1. Items for Modifiable Lifestyle Behaviors from Median Age 41 Data Collection}

\section{Smoking}

"And, a few questions about your smoking habits..."

20. Have you smoked at least 100 cigarettes in your life? (yes/no)

21. Do you smoke cigarettes now? (yes/no)

21a. How many cigarettes do you usually smoke per day

(\# cigarettes per day)

21b. How old were you when you started smoking cigarettes regularly?

(write in age, years old)

22. If you do not smoke cigarettes now, did you ever smoke cigarettes regularly?

(yes/no) continue if yes

22a. How old were you when you started smoking cigarettes regularly?

(write in age, years old)

22b. How many cigarettes did you usually smoke per day (\# cigarettes per day)

22c. How old were you when you stopped smoking cigarettes regularly?

(write in age, years old)

\section{Alcohol consumption}

"...And about your consumption of alcoholic beverages..."

30. Have you had more than 20 drinks in your entire life? (yes/no)

(continue if yes)

31. During the past 2 weeks, how many days did you drink any beer? (\# days)

31a. On the day(s) when you drank beer, about how many beers did you drink a day?

(\# beers a day)

32. During the past 2 weeks, on how many days did you drink any wine? (\# days)

$32 a$. On the day(s) when you drank wine, about how many glasses of wine did you drink a day? (\# glasses a day)

33. During the past 2 weeks, on how many days did you drink any hard liquor, such as whiskey, rum, gin, or vodka? (\# days)

33a. On the day(s) when you drank hard liquor, about how many drinks did you have a day? (\# drinks hard liquor a day)

\section{Physical Activity}

"...And now about your physical activity"

36. Do you usually participate in any of the following activities?

a. Climbing stairs instead of taking the elevator (yes/no)

b. Walking instead of driving short distances (yes/no)

c. Parking away from your destination so you have to walk more (yes/no)

d. Walking on your lunch break or after dinner (yes/no)

e. Getting off at a bus stop before your destination and walking (yes/no)

f. Other extra walking or stair climbing for exercise (yes/no) 
37. For at least the last three (3) months, which of the following activitites have you performed regularly?

a. Jog or run at least 10 miles per week (yes/no)

b. Play strenuous racquet sports (singles tennis, paddle ball etc) at least 5 hours

per week (yes/no)

c. Play other strenuous sports (basketball, soccer, etc) (yes/no)

d. Ride a bicycle at least 50 miles per week (yes/no)

e. Swim at least 2 miles per week (yes/no)

44. How many flights of stairs do you climb each day?

(write number, if zero put in "00". Let 10 steps=1 flight).

45. How many city blocks or their equivalent do you walk each day?

(Write in number, if zero put in "00." Let 1 mile=12 city blocks)

46. On average do you: (circle one)

1 Stroll at an easy pace

2 Walk at a normal pace

3 Walk fairly briskly

4 Walk fast (4 or more miles an hour)

\section{Social Engagement}

50. What is your current marital status (circle one)

1 Never married

2 Married once and married at present

3 Married once and terminated by death

4 Married once and terminated by divorce

5 Multiple marriage, married at present

6 Multiple marriage, not married at present

7 Separated

51. Do you regularly ( 2 or more times per month) participate in a church or religious group (yes/no)?

52. Are you an active member of a club, association, or organized activity in your community (yes/no)

53. Not counting your wife and other relatives, how many close friends do you have (friends that you feel at ease with, can talk to about personal matters and can call on for help.)

( $0=$ None $/ 1=1$ or $2 / 2=3$ to $5 / 3=6$ to $9 / 4=10$ or more)

54 . How many relatives, including your wife, do you feel close to? (relatives that you feel at ease with, can talk to about personal matters and can call on for help.)

( $0=$ None $/ 1=1$ or $2 / 2=3$ to $5 / 3=6$ to $9 / 4=10$ or more)

55 . How many of these close friends and close relatives, not counting your wife, 
do you see, write letters to or talk to on the telephone to at least once a month?

( $0=$ None $/ 1=1$ or $2 / 2=3$ to $5 / 3=6$ to $9 / 4=10$ or more)

\section{Diet}

"Let's begin with some questions about your food habits..."

1. How often do you usually eat the following (Circle one for each line)

(1=Daily; 2=3-6 times a week; 3=1-2 times a week; 4=1-3 times a month;

$5=$ less than once a month; $6=$ never)

a. Pork

b. Hot Dogs

c. Beef, Lamb

d. Poultry, veal

e. Eggs

f. Cold water fish (mackerel, salmon, sardines, bluefish, tuna)

g. Shell fish (Shrimp, lobster, crab)

h. Other fish

i. Dishes made of flour, cereal (dumplings, pancakes, spaghetti, macaroni etc)

j. Rice

k. Potatoes

I. Fruits and vegetables

m. Ice cream

n. Cheese

2. Estimate your daily consumption of the following: (Write in number per day)
a. Glasses of skim or low fat milk
b. Glasses of whole milk
c. Glasses of buttermilk
d. Cups of coffee
e. Cups of tea 
eTable 2. Frequencies of Early Midlife (Median Age 41) Covariates.

\section{Frequency Percent \\ (0=no/1=yes)}

Cardiovascular index

Depression

Diabetes

Liver Disease Index

Obesity (BMI >= 30)

Respiratory Index
0

1

0

1

0

1

0

1

385

70

84.62

15.38

\section{0}

1

0

443

10

97.79

2.21

416

92.44

34

7.56

455

99.34

3

0.66

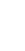

449

1

99.78

0.22

433

24

94.75

5.25

423

94.84

1

23

5.16

Note: $1=$ presence of disease/condition $0=$ absence of disease/condition; $\mathrm{BMI}=$ body mass index 
eTable 3. Correlations between Predictor Variables and Covariates.

PBAD

Age 20 AFQT (cognitive reserve)

p-value

Modifiable Lifestyle Behavior Composite

p-value

Education (lifetime max)

Ethnicity (1=non-Hispanic White/0=Other)

p-value

Stroke (VETSA 3; 1=yes/0=no)

Early Midlife (Age 40) covariates

Liver Disease (1=yes/0=no)

p-value

Respiratory Problems (1=yes/0=no)

p-value

Cardiovascular Problems (1=yes/0=no)

p-value

Depression (1=yes/0=no)

p-value

Diabetes $(1=$ yes $/ 0=$ no $)$

$p$-value

Obesity (1=BMI >=30/0=BMI < 30)

p-value

High Blood Pressure (1=yes/0=no)

p-value

Height (inches)

p-value
Early Adult

Cognitive

Reserve

0.192

$<.0001$

0.241

0.171

$<.0001$

0.155

0.345

0.266

0.001

$<.0001$

$-0.339$

$<.0001$

$-0.086$

$<.0001$

$-0.138$

$-0.074$

$-0.168$

0.002

0.100

0.000

$-0.025$

0.037

$-0.024$

0.599

0.439

0.615

0.019

0.052

$-0.086$

0.686

0.279

0.076

$-0.041$

$-0.043$

$-0.030$

0.381

0.361

0.532

$-0.012$

$-0.077$

$-0.121$

0.806

0.107

0.012

$-0.027$

$-0.022$

$-0.042$

0.571

0.644

0.380

0.028

0.049

$-0.021$

0.551

0.304

0.666

$-0.065$

$-0.051$

$-0.010$

0.165

0.280

0.829

0.101

0.088

0.020

0.023

0.050

0.675

Note: PBAD=Predicted Brain Age Difference Score; BMI=Body Mass Index 


\section{Supplemental Table 4. Correlations among Key Variables and LifeStyle Behaviors}

PBAD Lifestyle Composite Early Adult Cognitive Reserve

Stroke (by late midlife)

$\begin{array}{lr} & -0.138 \\ (p-v a l u e) & 0.002\end{array}$

0.125

( $p$-value)

0.719

0.009

Smoking

0.138

0.003

( $p$-value)

0.192

$<.0001$

(p-value)

$-0.073$

(p-value) $\quad 0.126$

0.127

0.007
0.064

0.190

$-0.168$

0.000

0.695

$<.0001$

0.525

$<.0001$

0.034

0.479

0.582

$<.0001$
0.232

$<.0001$

$-0.023$

0.636

0.035

0.465

0.069

0.146

Note: PBAD=Predicted Brain Age Difference Score. For lifestyle behaviors, higher scores indicate more favorable behaviors. 